\title{
Cytoplasm and cell motility Overview
}

\author{
M. Kirschner and K. Weber ${ }^{*}$
}

\begin{abstract}
Department of Biochemistry and Biophysics, University of California, San Francisco, California, USA and "Max Planck Institute for Biophysical Chemistry, Göttingen, Federal Republic of Germany
\end{abstract}

Current Opinion in Cell Biology 1989, 1:3-4

It is always difficult to look at recent history and predict the future. In this volume, however, it is unmistakably clear that the future course of cell biology is intimately tied to understanding the structure and organization of the cell, and that much of this understanding will be won on the battlefields of cytoskeletal research. There will be several important theaters of operations and several new technologies to be employed, but the overall goals are still the same as the original ones: the understanding of global features of cell behavior including cell movement, cell division, cell differentiation, and multicellular interactions in tissue formation.

The footsoldiers in these campaigns are still the major structural cytoskeletal proteins themselves. Though regulatory molecules and associated factors will modify their activities, many of the properties of the polymers will be determined by the intrinsic properties of the major subunits. It is clear that, unfortunately, we still don't understand clearly what these activities are. In the past few years the intrinsic dynamic properties of these simple structural polymers have been investigated by using new methodologies. The structural basis for dynamic instability and oscillations in microtubules remains an important area of study for both cell morphogenesis and mitosis. Yet, we still lack detailed structures for tubulin and its associated proteins. Intermediate filaments have become more dynamic and responsive and it is becoming clear that their remodelling is under cell cycle control, and subject to phosphorylation reactions that may regulate assembly. The beginnings of an understanding of how their overall morphology is regulated may be emerging with detailed studies of the interaction of intermediate filaments with other structural proteins. For actin, the evidence for dynamics in the cell is convincing, but the molecular basis has not yet been established. Just as the number of proteins that interact with actin appeared to be growing to an unmanageable level, the promise of in vivo tests of their function in genetically tractable systems like yeast and Dictyostelium has offered new hope.

The accumulation of ever increasing numbers of actinassociated and microtubule-associated proteins, though representing a triumph of immunocytochemical, protein chemical and more recently recombinant DNA methods, has seemed to outsiders, as well as a few insiders, to have degenerated into mere stamp collecting. In the past few years, however, these proteins have begun to segregate themselves into distinct prototypes carrying in their sequence casettes for different functions. The power of this approach has clearly emerged in the characterization of dystrophin, the gene product that is defective or missing in Duchenne-Becker muscular dystrophy. This protein, identified first by mapping and sequencing, is a large protein with an $\mathrm{N}$-terminal actin-binding domain followed by multiple triple-helical repeats first encountered in the erythrocyte protein spectrin. A further domain displaying EF hands has unknown function. Thus, dystrophin is unmistakably an actin binding protein; knowledge of the detailed structure of several actin binding proteins provided sufficient information to identify an unknown gene and to guess its function. The cytoskeletal field is now at a stage of maturity which allows the emergence of rules for structure and expression that have an impact on embryology and pathology. While antibodies and genetic lesions can now quickly yield protein sequence, it is only by laboring in the vineyards of cell biological research that we can now translate these structures into putative function.

Motility has always been the special province of the cytoskeletal field and this venue has been generally forsaken by biochemists who always felt uncomfortable about moving things (in general, they preferred to replace movement with ATPase assays). Biologists, as we all know, always liked creepy crawly things and the recent era has seen their triumph. Through direct visual assays, we have identified and purified both an anteriorgrade motor and a retrograde motor for microtubules; the latter is a cytoplasmic form of the dynein motor of cilia and flagella. Cilia and flagella themselves are fascinating as highly regulated organelles, and the recent ability to break down their structure and obtain partial motility reactions should allow for major advances in these areas.

As complicated as the cytoskeleton is, it is clearly not selfsufficient or independent. It is primarily a responsive element interpreting for example intrinsic signals for cell division and extrinsic signals for cell movement. The big questions of the mechanism of cell movement, chemotaxis, axon elongation and morphogenesis are still largely unanswered even though they were recognized as some of the biggest questions in biology almost a century ago. We first have the problem of linkage. What carries extra- 
cellular information to the responding cytoskeleton? Recent studies have identified a few types of receptor systems that can cross the plasma membrane with information that potentially could regulate the overall organziation of the cytoskeleton. Of particular importance are the $G$ proteins and the extracellular receptors, such as the integrins, cell adhesion molecules and the cadherins. Special adaptor molecules, perhaps like talin, must turn the extracellular information into intracellular information. Next we are faced with the problem of global organization. The leukocyte must not only move but it must move in a coherent way in response to only small differences in extracellular information. Here our information is still rather incomplete. Thus, an understanding of local interactions will not be enough to understand global features of cell organization.

Although it is tempting to plan the next decade of cytoskeletal research like a military campaign, such plans will undoubtedly suffer the fate of all long-range designs.

Most of the difficulties stem from our failure to take a wide enough view. The modern era in cytoskeletal research was itself born in exciting pictures of low-resolution that showed for the first time that structural pro teins spanned the cell in intricate and purposeful designs. The conservation of these proteins was unexpected, their extreme dynamics under many circumstances was un expected, their extreme heterogeneity was unexpected, and finally the conservation of domains of structure link- ing families of molecules such as the intermediate filament proteins and the nuclear lamins was also unexpected. There are further forks in the trail. After realizing that myosin was present in virtually all eukaryotic cells, it was natural to assume that it powered cell motility, as it powers muscle contraction. Recent experiments, however, have shown that most cell motility can occur when the expression or translation of the myosin gene is prevented. Our view of membrane trafficking may also be too narrow; our present extensive knowledge is mostly a product of considering the membrane systems separately from the cytoskeleton. Yet, recent methods of visualiz ing internal membranes in whole cells have enabled us to show both intimate interaction with the cytoskeleton and a morphology that suggests that cytoskeletal interactions could be an important determinant in membrane function.

There is no overall theme that emerges from the collection of essays in this issue, except that the cytoskeleton as a field is distinctly lacking in smugness. The un solved processes of cell behavior still dominate the thinking of even the most molecular of researchers. We have been rewarded by fascinating insights into protein design, new principles of the regulation of protein synthesis, novel physico-chemical mechanisms of assembly, and glimpses into evolution and pathology. However, we still face daunting challenges of understanding overall cell organization and behavior. 